\title{
Endoglycosidase expression in pubocervical fascia is up-regulated in menopause patients with severe pelvic organs prolapse
}

\author{
Svetlana V. Aidagulova \\ Novosibirsk State Medical University \\ Novosbirsk, Russia \\ s.aydagulova@gmail.com
}

\author{
Fedor A. Rakitin \\ Research Institute of Clinical and \\ Experimental Lymphology - \\ Branch ICG SB RAS \\ Novosibirsk, Russia \\ rakitinfedorr@mail.ru
}

\author{
Mikhail Yu. Soluyanov \\ Research Institute of Clinical and \\ Experimental Lymphology - \\ Branch ICG SB RAS \\ Novosibirsk, Russia \\ msoluyanov@mail.ru
}

\author{
Vadim V. Nimaev \\ Research Institute of Clinical and \\ Experimental Lymphology - \\ Branch ICG SB RAS \\ Novosibirsk, Russia \\ nimaev@gmail.com
}

\author{
Igor O. Marinkin \\ Novosibirsk State Medical University \\ Novosbirsk, Russia \\ rectorngmu@yandex.ru
}

\begin{abstract}
Pelvic organs prolapse in women is very inconvenient age-dependent pathology. One of the causes of pelvic organs prolapse is considered so called undifferentiated dysplasia of connective tissue being genetically determined change in the morphogenesis of connective tissue. The aim of this work was to study heparanase expression in pubocervical fascia of menopause women with pelvic organ prolapse. As shown heparanase protein content was significantly upregulated in cellular compartment of pubocervical fascia in patients with pelvic organs prolapse and prominent undifferentiated dysplasia of connective tissue degrees.
\end{abstract}

Keywords - pelvic organs prolapse, undifferentiated dysplasia of connective tissue, endoglycosidase, pubocervical fascia, menopause women

\section{Motivation and Aim}

Pelvic organs prolapse (POP) in women is very inconvenient age-dependent pathology. The incidence of women with POP in USA and other countries is reaching up to $50 \%$ in patients aged 80 years [1]. The understanding of POP's pathophysiology is important for prevention and treatment. Presumably POP deals with so called undifferentiated dysplasia of connective tissue (UCTD). UCTD is genetically determined change in the morphogenesis of connective tissue due to abnormalities of fibrillogenesis and non-fibrillar extracellular matrix (ECM). In POP it was known the expression changes mainly focusing on the genes/proteins of collagen, elastin, matrix metalloproteinases (MMPs) and their tissue inhibitors [2]. Besides MMPs the ECM also contains at least one's more enzyme involved in connective tissue remodeling, which has an endoglycosidase activity and is named heparanase (HPSE). The aim of this work was to study HPSE expression in pubocervical fascia of menopause women with POP.

\section{Methods and Algorithms}

This study was performed on 150 women $60,7 \pm 7,73$ years old with POP, the diagnosis was based on the POP Quantification (POP-Q ISC, 1996). The average duration of menopause was $10,9 \pm 9,1$ years. Women with malignances and/or active/latent urinary tract infection were excluded. All 150 patients were suitable for POP-Q III-IV (Ba) and underwent primary scheduled treatment using vaginal access surgery; also in all cases the informed consent of the patient to the examination and treatment of POP was taken in accordance with the directives of the European Community (86/609 / EEC) and the Helsinki Declaration, in compliance with the «Ethical Principles for Scientific Medical Research with Human Participation» and in accordance with the «Rules of Clinical Practice in Russian Federation». Patients were divided into 2 representative by age and by POP's severe groups, depending on the UCTD degree. The 1st group consisted of 48 women with mild degree of UCTD. The 2 nd group consisted of 102 women with moderate and severe degree of UCTD. Phenotypic manifestations of UCTD were evaluated on the basis of physical examination and ultrasound study of the heart and pelvic organs, focusing on pathological renal mobility. The minimal manifestations of UCTD included joint hypermobility, mild skin striae and/or myopia. Their combination with signs of the «MASS phenotype» (mitral valve prolapse, additional heart chords, scoliosis, and so on) were interpreted as moderate and severe degree of UCTD [3]. Surgical samples of the pubocervical fascia were fixed in $10 \%$ buffered formalin solution. For immunohistochemistry, 5-mkm sections of formalin-fixed, paraffin-embedded tissue sections were deparaffinized and antigens were retrieved in sodium citrate buffer $(10 \mathrm{mM}$ sodium citrate, $0,05 \%$ Tween-20) at $95-98^{\circ} \mathrm{C}$ for $20 \mathrm{~min}$. The rabbit polyclonal anti-HPSE (Abcam, cat. № ab85543,1:100) primary antibodies were used. For negative control, the primary antibodies were replaced by $5 \%$ bovine serum. HPSE-immunostaining patterns were visualised using Histostain-Plus 3rd Gen IHC Detection Kit (ThermoFisher Scientific). The sections were counterstained with Hematoxylin and observed by light microscopy using Axio Scope.A1 microscope with the camera AxioCam MRc5 and software ZEN blue for the quantitative analysis of 30 images per group with magnification 40x10 (Zeiss, Oberkochen, Germany). Statistical processing of the results was performed using the statistical software application package STATISTICA v.6.0. A value of $p<0,05$ was considered to indicate a statistically significant difference. 


\section{Results}

An analysis of the incidence of clinically polymorphic symptoms and diseases inherent to the UCTD in 150 women of 1 st and 2 nd groups have revealed the genital prolapses and hernia as universal UCTD manifestations in first-line relatives of $40 \%$ and $49 \%$ cases, respectively. In women of the 2 nd group, varicose veins occurred in 79,4\% comparing 33,3\% cases in the 1st group. And in contrast to the 1st group, in the 2 nd group, the biliary dyskinesia was detected in $45 \%$ of women, and heart rhythm and conduction disturbances were revealed in $48 \%$ ones. The investigation a hypothesis on a potential negative effect of HPSE content on the POP pathogenesis, the dense fibrous connective tissue of pubocervical fascia was studied with emphasis on the intraand extracellular endoglycosidase localization. HPSE protein content was significantly up-regulated in cellular compartment of pubocervical fascia of the 2nd group with prominent UCTD degrees compared with the 1st one: the average area of total positive immunostaining products amounted as 2,4 $\pm 0,6 \mathrm{~mm} 2$ versus $1,3 \pm 0,5 \mathrm{~mm}^{2}(\mathrm{p}<0,01)$.

\section{Conclusion}

HPSE known as heparan sulfate cleaver and active participant in different pathological processes such as inflammation and tumorigenesis may affect the ECM properties of pubocervical fascia and should serve as new molecular marker of UCTD and POP.

\section{References}

[1] Nygaard I. et al. (2008) Prevalence of symptomatic pelvic floor disorders in US women. JAMA. 300: 1311-1316.

[2] Lim V.F. et al. (2014) Recent studies of genetic dysfunction in pelvic organ prolapse: the role of collagen defects. Aust N Z J Obstet Gynaecol. 54: 198-205.

[3] Smolnova T.Yu. et al. (2003) The phenotypical symptom complex of connective tissue dysplasia in females. Clin. Med. 8: 42-47. 